\title{
"Wolkige Raumforderung mit einer hühnereigroßen Dimension“
}

\section{ICIS-Tagung 2017: Aktuelle Themen und Innovationen in der onkologischen Bildgebung}

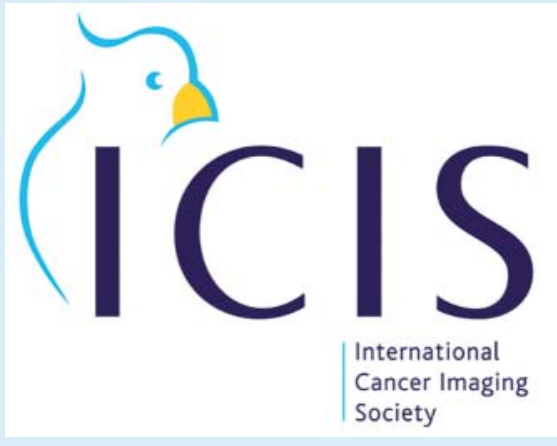

Zum zweiten Mal nach 2014 findet die Tagung der International Cancer Imaging Society (ICIS) in Deutschland statt. Vom 2. bis 4. Oktober 2017 kommen Experten aus dem In- und Ausland im Langenbeck-Virchow Haus in Berlin zusammen, um sich ausschließlich mit Bildgebung und radiologischen Interventionen bei onkologischen Patienten zu beschäftigen. Prof. Dr. Stefan Diederich, Präsident der ICIS, gewährt im Interview interessante Einblicke in die onkologische Bildgebung und stellt die Höhepunkte der Tagung vor.

Nach Informationen des Robert Koch Instituts leben in Deutschland rund vier Millionen Menschen, die schon einmal an Krebs erkrankt sind. Rund $\mathbf{5 0 0 0 0 0}$ Krebsfälle werden jedes Jahr neu gemeldet, 220000 Menschen sterben Jahr für Jahr an dieser Erkrankung. Was Iösen solche Zahlen in Ihnen aus?

In der westlichen Welt gibt es zwei Erkrankungsgruppen, die überdurchschnittlich häufig zum Tod führen. Die eine Gruppe sind die Herz-Kreislauf-Erkrankungen, die andere umfasst Erkrankungen an Krebs. Alle anderen Krankheiten verlaufen deutlich seltener tödlich, was sicherlich auch auf entsprechende Fortschritte in der Medizin zurückzuführen ist. Heute stirbt man - zumindest in unserer Hemisphäre - nicht mehr an einer banalen Lungenentzündung. Deshalb werden Herz-Kreislauf-Erkrankungen und Krebs quantitativ wichtiger. Die vorgestellten Zahlen sind nicht schön, aber für mich persönlich auch kein Grund für Frustration. Selbstverständlich haben wir
Ärzte den Anspruch, mit den uns zur Verfügung stehenden diagnostischen und therapeutischen Mitteln eine Heilung herbeizuführen. Beschwerden zu lindern und qualitative Lebenszeit zu ermöglichen sind jedoch ebenfalls Ziele, die vielleicht nicht gleichwertig sind, aber dennoch eine nicht zu unterschätzende Rolle spielen. Ich begleite viele Krebspatienten, bei denen ich weiß, dass sie mit großer Wahrscheinlichkeit an der Erkrankung sterben werden. Durch eine gute Diagnostik, die dem Onkologen oder Strahlentherapeuten bei der Wahl der bestmöglichen Behandlung hilft, trage ich jedoch entscheidend dazu bei, dass der Patient die ihm verbleibende Zeit mit einer guten Lebensqualität ausfüllen kann. Teilweise kann ich selbst durch minimal-invasive Eingriffe unmittelbar das Leid eines Patienten lindern und für einen Zugewinn an Lebensqualität sorgen. Auch das ist ein Wert an sich. Die entscheidende Frage heute ist nicht so sehr die nach dem Lebensalter, sondern vielmehr die, mit welcher Lebensqualität jemand alt wird. Dies gilt für Krebspatienten genauso wie für jeden anderen Patienten. Außer Frage steht jedoch, dass Krebs für viele Menschen ein Schreckgespenst ist. Auch ich habe vor bestimmten Krebssorten Angst, was dazu führt, dass ich mich mit meinem Leben bewusster auseinandersetze. Was ist gut, was bedeutet mir viel, was möchte ich auf jeden Fall noch machen und deshalb auch nicht auf die lange Bank schieben? Krebs ist also auch etwas, dass das Leben bewusster und lebenswerter machen kann und für mich persönlich natürlich auch deshalb ein großes Thema, weil ich in einem Krankenhaus arbeite, in dem sich sehr viel um Krebs dreht.

\section{In regelmäßigen Abständen liest man in den Medien von dem großen Durchbruch bei der Behandlung von Krebs. Aber Ärz- te können auch heute mit der Operation, Chemotherapie und Bestrahlung nur die klassischen Drei anbieten oder?}

Dies sind immer noch die zentralen Säulen der Therapie, die je nach Krebsart, Stadium und Organbefall einen sehr unterschiedli-

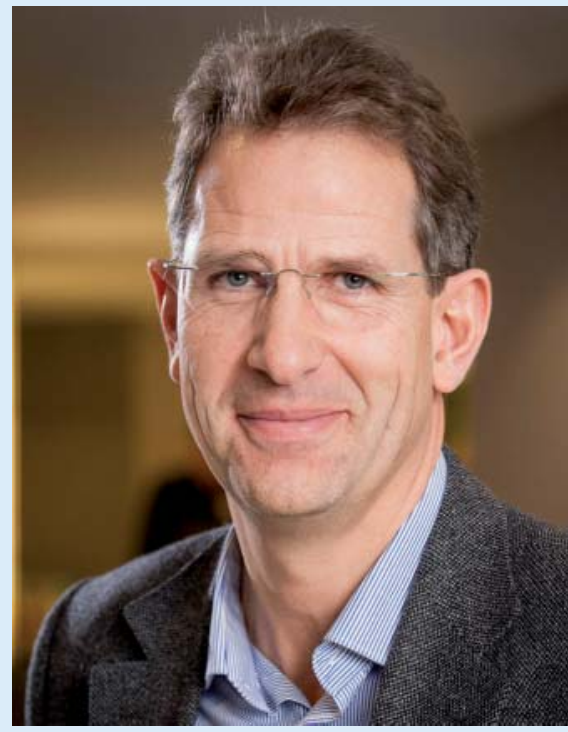

Prof. Dr. Stefan Diederich

chen Stellenwert haben. Das war so und wird sich in absehbarer Zeit auch nicht dramatisch ändern. Auf der anderen Seite entwickeln die Strahlentherapeuten immer bessere Konzepte, um eine hohe Strahlendosis in den Tumor zu bekommen und dies bei einer gleichzeitig möglichst geringen Strahlendosis für das anliegende gesunde Gewebe. Die medikamentöse Therapie hat ebenfalls große Fortschritte gemacht und ist heute mehr als die klassische Chemotherapie. Mittlerweile gibt es Antikörpertherapien, sogenannte small molecules oder auch ganz neue Immuntherapien, bei denen das Medikament gar nicht gegen den Krebs gerichtet ist, sondern auf das Immunsystem so wirkt, dass es den Krebs bekämpft. Auch die Chirurgen haben sich weiterentwickelt. Die Operationstechniken von heute sind für den Patienten deutlich schonender und sicherer. Es ist und bleibt ein besonders spannendes Feld in der Medizin. Wir lernen fast täglich mehr über den Krebs, was sich auch auf die Diagnostik und Therapie auswirkt. Das ist übrigens auch ein zentraler Grund dafür, weshalb Tagungen und Kongresse wie ICIS 2017 so wichtig sind. Gerade auf dem Gebiet der Onkologie und der onkologischen Bildgebung tut sich in kurzer Zeit so unglaublich viel, dass es für den Einzelnen sehr schwer 
ist, da auf dem Laufenden zu bleiben. Ein Kongress, der alle aktuellen Entwicklungen zusammenfasst und vermittelt, stellt deshalb einen sehr guten Weg dar, den bestehenden Informationsbedarf auch abzudecken.

Die Überlebensaussichten bei einer Krebserkrankung haben sich in Deutschland innerhalb der letzten $\mathbf{3 0}$ Jahre deutlich verbessert. Welchen Anteil hat daran die onkologische Bildgebung?

Das Problem ist, dass man die Bedeutung der Bildgebung in vielen Bereichen kaum quantifizieren kann. Wenn eine neue Therapie eingeführt wird, beschäftigt sich die Forschung vorrangig mit der Frage, ob damit die Überlebensrate der Patienten im Vergleich zur alten Therapie gestiegen ist. Derartige Untersuchungen gibt es für die Chemotherapie und andere medikamentöse Therapien, für Strahlentherapien und für chirurgische Verfahren. Es gibt aber sehr wenig Forschung darüber, welchen Einfluss auf das Überleben eines Patienten die Bildgebung hat bzw. die Entscheidung darüber, wann welche bildgebende Methode eingesetzt wird und ob dies in einem High Volume Center geschieht, in dem das Expertenwissen gebündelt ist, oder aber in einer anderen Einrichtung, in der der durchführende Radiologe tagtäglich ganz unterschiedliche Fragestellungen beantworten muss. Ich persönlich bin der Ansicht, dass die Bildgebung eine herausragende Rolle spielt, wenn es darum geht, die Heilungschancen von Krebspatienten zu erhöhen oder aber die bestmögliche Lebensqualität sicherzustellen. Nehmen sie als Beispiel den Bauchspeicheldrüsenkrebs. Bei diesem Krebs ist auch 2017 in der Regel die einzige realistische Chance auf Heilung eine Operation. Früher hat man sehr viele Patienten exploriert, d.h. sie wurden operiert, um nachzuschauen, ob der Tumor entfernt werden konnte oder nicht. Wenn ja, wurde er entfernt und der Patient hatte damit seine Heilungschance. Wenn der Tumor nicht operabel war, wurde der Bauch einfach wieder zugemacht. In diesem Fall war die Operation eigentlich überflüssig und für den Patienten nur mit Schmerzen, Risiken und Belastungen verbunden. Das kann man heute durch moderne Bildgebung, speziell durch MRT und CT, in den meisten Fällen vermeiden. Für den Patien- ten bedeutet dies zwar keine messbar bessere Heilungschance, aber sicherlich ein Zugewinn an Lebensqualität, da er die verbleibende Zeit ohne die negativen Begleiterscheinungen einer Operation verbringen kann. Mithilfe der Bildgebung kann ich auch die Frage beantworten, ob bereits Fernmetastasen vorliegen. Denn wenn ich eine derart aufwändige Operation durchführe und es stellt sich erst dann heraus, dass der Patient Fernmetastasen hat, war alle Mühe vergebens. Und wenn er umgekehrt fälschlich als metastasiert eingeordnet und deshalb nicht operiert wird, verliert der Patient ebenfalls. Aber wie gesagt, leider haben wir kaum große multizentrische, randomisierte Studien, die den konkreten Nutzen von Bildgebung aufzeigen würden, unabhängig von den weiteren Therapieschritten. Studien beurteilen in der Regel den gesamten Prozess von der Diagnose bis zur Therapie.

Wie bewerten Sie persönlich denn vor dem Hintergrund des Gesamtspektrums onkologischer Therapien die Relevanz und auch Akzeptanz der zur Verfügung stehenden minimal-invasiven Verfahren der interventionellen Onkologie?

Die Akzeptanz auf Seiten des Patienten ist eigentlich immer gegeben, weil die minimal-invasiven Verfahren der interventionellen Onkologie genau dem entsprechen, was er sich wünscht - kleiner Schnitt, kleiner Schmerz, kleines Risiko. Oft werden allerdings diese Verfahren trotzdem unterschätzt, da viele minimal-invasive Eingriffe unter Vollnarkose durchführen werden. Die Akzeptanz in anderen Fächern stellt sich nicht automatisch ein. Gerade in den Bereichen, in denen die Kenntnisse über radiologische Interventionen nicht ausgeprägt sind, wird die Intervention erst einmal als eine Konkurrenz empfunden, die man eigentlich nicht haben möchte. Je mehr man sich jedoch miteinander beschäftigt, desto schneller löst sich diese Distanz nach meiner Erfahrung in Wohlgefallen auf. Es ist im Grunde auch ganz einfach: Wenn man über ein breites therapeutisches Angebot verfügt, hat der Patient eher das Gefühl, dass er das, was sinnvoll erscheint, auch tatsächlich angeboten bekommt. Wenn jedoch bestimmte Verfahren gar nicht angeboten werden, spricht sich dies schnell herum und hat mitunter Auswirkungen auf die Patientenströme insgesamt. Ich habe wiederholt erlebt, dass bei Chirurgen anfangs die Angst groß war, dass der Radiologe dem Chirurgen seinen Eingriff wegnimmt. Das war beispielsweise so bei der Embolisation von Uterusmyomen. Einige Gynäkologen haben befürchtet, dass sie ihre operativen Myom-Therapien verlieren würden. Das Gegenteil war der Fall. Frauen, die wussten, dass an einer Einrichtung die Myom-Embolisation angeboten wird, haben sich gezielt dorthin gewandt. Ein wesentlicher Teil wurde dann doch nicht mit einer Embolisation behandelt und hat damit die Operationszahlen der Gynäkologie nach oben getrieben. Ein anderes Beispiel ist der Aortenstent. Die Gefäßchirurgen waren anfangs sehr besorgt, dass die Stents in radiologischer Hand die operativen Eingriffe an den Aneurysmen abschaffen würden und die Gefäßchirurgie damit nichts mehr zu tun hätte. Aber auch hier zeigte sich, dass die Patientenströme größer wurden, weil beides, die Operation und die Intervention, angeboten wurden. Am Ende hatten Radiologen und Gefäßchirurgen mehr als vorher zu tun. So ist es auch in der Onkologie. Hier sitzt man ohnehin im Tumorboard zusammen und bespricht gemeinsam, wie nun mit dem Patienten weiter zu verfahren ist. So kann es sein, dass der Chirurg erst einmal operieren soll, um das zu entfernen, was operativ entfernt werden kann. Und dann bleibt vielleicht eine Metastase übrig, die man chirurgisch nicht gut behandeln kann. Die wird dann minimal-invasiv durch den Radiologen abgetragen. Oder es gibt einen Patienten mit einem Tumor, der inoperabel aussieht, aber wenn dieser mit einer Chemoembolisation vorbehandeln wird, bekommt man ihn möglicherweise in der Größe so reduziert, dass er dadurch operabel wird. Ein anderes Beispiel: Ein Patient hat multiple Metastasen. Alle werden infolge der Chemotherapie kleiner, bis auf eine, die stattdessen wächst. Was kann man tun? Der Onkologe müsste seine gesamte Chemotherapie umstellen in der Hoffnung, dass neue Substanzen alle Metastasen treffen. Er sucht sich aber in der Regel seine Medikamente danach aus, welche die beste Kombination aus Wirkung und Nebenwirkung aufweisen. Wenn er also auf eine Second-Line-Chemotherapie setzt, wäre das Risiko groß, dass entweder der Effekt weniger gut ist oder sich der gewünschte 
Effekt zwar einstellt, aber die Nebenwirkungen deutlich höher sind. Die sinnvolle Alternative wäre, die eine Metastase, die nicht gut auf die Chemotherapie angesprochen hat, mit einer Thermoablation zu behandeln. Dies kann ohne Unterbrechung der eigentlich wirksamen Systemtherapie geschehen. Für einen chirurgischen Eingriff müsste hingegen die Chemotherapie für einige Wochen unterbrochen sein. Insofern ergänzen sich die Methoden eher als das sie in Konkurrenz zueinander stünden.

Es gibt bis zu 300 Krebsarten und Subtypen, wobei 500000 neue Krebsfälle jährlich auch für 500000 neue, individuell unterschiedliche Tumorgeschichten stehen. Eine Antwort darauf lautet individualisierte Tumortherapie. Inwieweit verändert sich damit Rolle der Bildgebung?

Damit einher geht sicherlich ein weiterer Bedeutungszuwachs der Bildgebung. Aber es wird auch komplizierter. Individualisierte Medizin bedeutet ja, dass der einzelne Patient nicht mehr in eine Schublade gesteckt wird, auf der z. B. Brustkrebs steht, und dann einer Einheitstherapie zugeführt wird. Es gibt harmlose Varianten, die, lange unentdeckt, kaum wachsen und bei denen dann zum Zeitpunkt der Diagnose trotzdem kein Lymphknotenbefall oder eine Fernmetastasierung vorliegt. Zum Brustkrebs gehört aber auch der $1 \mathrm{~cm}$ große Tumor einer jungen Frau, der bereits zu einem sehr frühen Diagnosezeitpunkt Fernmetastasen aufweist. Dieser Tumor ist biologisch eigentlich nicht verwandt mit dem vorher beschriebenen, langsam wachsenden Tumor. Solche Facetten hat auch der Lungenkrebs, der im Prinzip sehr bösartig ist. Rein statistisch überleben nur ca. 15 Prozent der Patienten ihren Tumor um fünf Jahre oder länger. Aber auch hier kann es sein, dass ein Tumor Jahre braucht, um erkennbar zu wachsen, dann diagnostiziert wird, keine Fernmetastasen aufweist, und der Patient nach einer operativen Entfernung als geheilt gilt. Diese ganz unterschiedlichen Ausprägungen können mithilfe der Bildgebung teilweise frühzeitig klassifiziert werden, sodass auch eine Prognose getroffen werden kann, um welchen Tumortypen es sich im konkreten Fall handelt. Es gibt spannende Ansätze im MRT, über Diffusionswichtung, d. h. über die
Berechnung der apparent diffusion coefficient (ADC) values Aussagen über die biologische Aggressivität eines Tumors zu treffen. Es gibt die Perfusions-CT, die Erkenntnisse über die Durchblutung eines Tumors liefert. Die Art und Weise, wie hier der Tumor das Kontrastmittel aufnimmt, kann wichtige Hinweise geben, wie bösartig er in dem jeweiligen Organ sein wird. Die Bildgebung ist auch entscheidend bei der Frage, ob ein Tumor vorbehandelt werden soll, z. B. mit einer Chemotherapie, einer Bestrahlung oder auch beidem. Es gibt für bestimmte Tumoren Indizien, die darauf hinweisen, ob sie auf so eine neoadjuvante Therapie ansprechen werden oder nicht. Die Vorhersage, ob eine Vorbehandlung erfolgreich sein wird, ist ein ganz spannendes Thema beim Konzept der individualisierten Tumortherapie. Die Bildgebung spielt aber auch bei der Verlaufskontrolle eine immer wichtigere Rolle. Traditionell erfolgte eine Verlaufskontrolle in relativ großen Zeiträumen und orientierte sich allein an der Größe des Tumors. Eine Chemotherapie beispielsweise musste den Tumor bzw. die Krebszellen zunächst zerstören. Diese toten Zellen wurden im Körper dann abgebaut und erst nach diesem Abbau war dann im positiven Fall ein Schrumpfungsprozess bildgebend erfassbar, d. h. der Tumor war im Durchmesser kleiner geworden. Mit den neuen Methoden, also Perfusions-CT und MRT, Messung des ADC-Wertes oder auch PET-CT, kann man schon sehr früh, nach einer Woche oder im günstigsten Fall schon nach einem Tag eine Aussage darüber treffen, ob der Tumor auf eine Therapie anspricht. Dieser Beurteilung liegen ganz andere Eigenschaften des Tumors zugrunde. Die PET-CT kann man z. B. bei Gastrointestinalen Stromatumoren, Weichgewebstumoren, die im Magen-Darmtrakt vorkommen, einsetzen. Für diese Tumoren gibt es mit den Tyrosinkinase-Inhibitoren neue Medikamente, die den Zellstoffwechsel blockieren. Gastrointestinalen Stromatumoren verstoffwechseln nämlich sehr intensiv Glukose, was man durch eine PET-CT mit „F-2-Fluor-2-deoxyD-glucose“ (FDG) als Radiotracer darstellen kann. Bereits nach der ersten Medikamentengabe, die oral in Tablettenform erfolgt, kann so nachgewiesen werden, ob der Glukose-Stoffwechsel des Tumors dadurch zusammengebrochen ist oder nicht. Das ist für mich ein Paradebeispiel für eine indivi- dualisierte Therapie, bei der durch Bildgebung zu einem sehr frühen Zeitpunkt die Wirksamkeit einer gewählten Therapie beurteilt werden kann. Damit bietet sich auch die Möglichkeit, Therapien, die nachweislich keine Veränderung herbeiführen, auch sehr früh wieder abzusetzen. Das nützt nicht nur dem Patienten, der von etwaigen Nebenwirkungen verschont bleibt, sondern auch unseren Sozialsystemen, die dadurch finanziell entlastet werden. Schließlich sind viele der modernen individualisierten Therapien auch sehr teuer. Eine Verlaufskontrolle wie oben beschrieben funktioniert nicht bei allen Tumoren. Das macht es für uns Radiologen sehr kompliziert, denn den einen Standardtest, der mir nach drei Tagen sagt, ob eine Therapie wirkt, gibt es nicht. Deshalb ist auch ein Kongress wie das ICIS-Meeting so wichtig. Hier werden möglichst viele Facetten und Ausprägungen thematisiert.

Darmkrebs, Lungenkrebs, Hautkrebs, Brustkrebs und Prostatakrebs machen gut die Hälfte der Neuerkrankungen aus. Finden diese Krebsarten eine entsprechende Berücksichtigung im Programm der ICIS-Tagung?

Ja und Nein. Die Themen, die Radiologen besonders beschäftigen, finden sich auch in einer entsprechenden Gewichtung im Kongressprogramm wieder. Dabei handelt es sich aber nicht um eine „eins zu eins Übersetzung“ der Neuerkrankungszahlen. Ein Thema, das auf der ICIS-Tagung in diesem Jahr einen relativ großen Stellenwert einnimmt, ist das Prostatakarzinom. Der Grund dafür ist schlicht der, dass hier in der Bildgebung gerade sehr viel passiert. Auf der anderen Seite hat die ICIS-Tagung auch den Anspruch, onkologische Bildgebung in ihrer Breite abzudecken. Es gibt deshalb auch Sitzungen zu Nischenthemen wie z. B. Krebs bei Kindern. Das kommt glücklicherweise zwar selten vor, aber wenn der Fall einmal eintritt, ist das natürlich eine große Katastrophe für das Kind und die Familie. Im Programm haben wir deshalb Sitzungen, in denen Kinderradiologen über die onkologische Bildgebung und onkologische Interventionen reden. Wir haben auch eine Sitzung zu Kopf- und Halstumoren. Das sind zwar eher selten auftretende Tumoren, aber für die Betroffenen deshalb nicht minder bedeutsam und ein- 
schneidend. Es geht uns auch darum, mit dem Programm alle Regionen des Körpers abzudecken, bei denen die Bildgebung Relevanz hat. Sicherlich finden sich dort Tumoren, die häufiger auftreten, auch überproportional wieder, aber auch seltener auftretende Erkrankungen sind wichtige Themen, damit diejenigen, die ein besonderes Interesse an onkologischer Bildgebung haben, auch für diese Fälle gerüstet sind. Wir wollen mit der ICIS-Tagung ja nicht nur die Spezialisten der onkologischen Bildgebung ansprechen, sondern auch den Allgemeinradiologen, der vielleicht morgens eine Schulter-MR befunden muss und nachmittags dann bei einem Kolonkrebs-Patienten ein CT-Staging vornimmt.

Welches sind aus Ihrer Sicht besonders interessante Entwicklungen, die sich auch im ICIS-Programm widerspiegeln?

Ein ganz spannendes Thema ist die Immuntherapie. Hier werden Medikamente eingesetzt, die den Krebs nicht direkt attackieren, sondern das körpereigene Immunsystem so stärken, dass es seinerseits den Krebs angreifen kann. Wichtig dabei ist, dass diese Medikamente im Patienten andere Effekte auslösen können als man sie von einer klassischen Chemotherapie kennt. Eine wirksame Chemotherapie macht den Tumor kleiner, eine erfolgreiche Immuntherapie hingegen kann den Tumor unter Umständen erst einmal größer machen. Der Radiologie, der diese Patienten untersucht und befundet, muss wissen, dass gerade zu Beginn der Immuntherapie der Tumor durchaus erst einmal schwellen darf, da Entzündungszellen aus dem Immunsystem in den Tumor einströmen, um ihn dann zu zerstören. Diesem Thema widmen wir auf der ICIS-Tagung sogar eine Key Note Lecture. Ein anderes zentrales Thema ist die Response-Beurteilung. Woran erkenne ich, dass ein Tumor auf eine Therapie anspricht? Es ist, wie bereits gesagt, nicht mehr alleine eine Frage der Tumorgröße. Wir können heute die Perfusion des Tumors beurteilen, den GlukoseStoffwechsel erfassen, oder Änderungen in der Binnenstruktur erkennen. Im IT-Bereich gibt es Entwicklungen, die in der Radiologie zunehmend an Bedeutung gewinnen. Hierzu zählen insbesondere die strukturierte
Befundung und das maschinenbasierte Lernen. Wir haben Sitzungen in das Programm aufgenommen, die Radiologen dabei unterstützen sollen, nicht länger Prosatexte zu formulieren, in denen in unterschiedlichsten Begrifflichkeiten ein Tumor beschrieben wird, z. B. „wolkige Raumforderung mit einer hühnereigroßen Dimension“. Es geht vielmehr um eine Fokussierung auf die für die weiteren Therapieschritte relevanten Informationen, d. h. exakte Größe, Randbegrenzung, Organbezüge zu Nachbarstrukturen, Kontaktflächen zu bestimmten Organen. Eine weitere Frage lautet, ob mir ein Computerprogramm mithilfe von eingespeisten großen Datensätzen zukünftig eine Liste von in Frage kommenden Differenzialdiagnosen anbieten kann oder eine Prognose darüber abgibt, wie hoch die Wahrscheinlichkeit einer Bösartigkeit des jeweiligen Tumors ist. Es gibt beispielsweise erste konkreten Ansätze, bei denen ein Computerprogramm für einen Lungenrundherd, den man - unter Kenntnisnahme des Alters des Patienten und seiner Rauchgewohnheiten - nach Größe, Dichte, Begrenzung beschrieben hat, einen Prozentwert errechnet, ob dieser Herd maligne sein wird. Diese Entwicklungen werden den Radiologen sicherlich nicht ersetzen, können ihn aber bei der Befundung wirksam unterstützen. Schließlich passiert auch im Bereich der onkologischen Interventionen gerade sehr viel. Das ist nicht zuletzt deshalb auch ein besonderes Schwerpunktthema auf der ICIS-Tagung. Die perkutane Tumortherapie wie auch die transvaskuläre Tumortherapie sind Verfahren, die eine möglichst effektive Behandlung mit einer bestmöglichen Lebensqualität des Patienten verbinden. Wenn die Möglichkeit gegeben ist, statt einer großen Operation unter Vollnarkose und einem mehrtägigen Aufenthalt auf der Intensivstation eine Intervention unter radiologischer Kontrolle, mit einer örtlichen Betäubung oder einer leichten Beruhigungsspritze durchzuführen und damit den gleichen Effekt zu erzielen, dann mag dies vielleicht keine signifikanten Auswirkungen auf die Heilungschancen des Patienten haben, sehr wohl aber auf seine Lebensqualität. Es gibt also viele interessante Entwicklungen, die wir auch im Tagungsprogramm abbilden werden.
Sie haben in diesem Jahr neben klassischen Vorträgen eine ganze Reihe praxisorientierter, interaktiver Veranstaltungsformate geplant. Welche sind das genau und was versprechen Sie sich davon?

Auf radiologischen Kongressen ist schon seit längerem die Tendenz erkennbar, mehr fallorientiert zu lernen und nicht Frontalvorträge, die Lehrbuchwissen vermitteln, in den Vordergrund zu stellen. Diese haben durchaus ihre Berechtigung, weil sie gerade für Allgemeinradiologen ein passendes Format sind, um sich viele Informationen in relativ kurzer Zeit anzueignen. Irgendwann stößt man aber mit dem reinen Frontalunterricht auch an Grenzen. Auf der ICIS-Tagung haben wir deshalb, neben reinen Vorträgen, insgesamt acht Hands on-Workshops zu acht verschiedenen Themen, in denen relativ kleine Gruppen von bis zu 40 Personen mit zwei Tutoren an PACS-Befundungs-Workstations Fälle aus der radiologischen Praxis durchgehen. Die Tutoren werden kurz in das jeweilige Thema einführen, um dann den Teilnehmern die Gelegenheit zu geben, in Zweier- oder Dreier-Gruppen die aufgerufenen Fälle zu analysieren und mit den Tutoren zu diskutieren. Idealerweise berichten die Tutoren dann im Anschluss über den tatsächlichen Therapieverlauf. Noch praxisnäher wird es bei den Live-Cases, die wir in diesem Jahr zum ersten Mal anbieten und bei denen es ausschließlich um onkologische Interventionen gehen wird. Die Gruppen sind hier mit maximal acht Personen noch einmal deutlich kleiner. Die Teilnehmer bekommen Gelegenheit, in der Charité und in der evangelischen Lungenklinik bei speziellen Interventionen live dabei zu sein. In einem ersten Schritt wird hier der Fall vorgestellt und der Entscheidungsfindungsprozess im Tumorboard rekapituliert. Dann werden die Teilnehmer tatsächlich daneben stehen, wenn der Patient behandelt wird - beispielsweise mit einer Thermoablation oder eine Katheter-Intervention. An diesem Punkt wird es wirklich ganz praktisch. Mit welchem Material wird gearbeitet, welche Hindernisse werden erwartet, welche Möglichkeiten gibt es, die Hindernisse zu umschiffen. Die Kunst der Intervention besteht ja nicht im Setzen der Nadel oder eines Katheters. Sie besteht vielmehr darin, sich für die richtige Maßnahme zu entscheiden, Hindernisse zu erkennen und zu wissen, wie 
man sie umschiffen kann, oder Komplikationen frühzeitig wahrzunehmen und zu beherrschen. Darüber entlang eines konkreten Falls mit jemandem, der das regelmäßig macht, sprechen zu können, hat noch einmal einen ganz anderen Lerneffekt als ein Vortrag, der idealtypische Verläufe vorstellt und bei dem die Bilder immer perfekte Befunde zeigen. Am Ende der Live Cases besteht auch die Möglichkeit zur Manöverkritik. Was hat gut geklappt, was hätte man retrospektiv vielleicht anders machen sollen, sind noch Folgeeingriffe zu erwarten, wie würde man den Patienten kontrollieren? Die Teilnehmer werden also an einem speziellen Fall von der Indikationsstellung bis zur Nachsorge alles erleben.

Für die ICIS-Tagung haben Sie auch Stipendien ausgelobt. Wer kann sich dafür bewerben?

Es handelt sich dabei um Stipendien der Deutschen Röntgengesellschaft, die allgemein für internationale Kongresse wie jetzt auch die ICIS-Tagung speziell an junge Radiologen vergeben werden. Voraussetzung für eine Bewerbung ist die Mitgliedschaft in der DRG. Außerdem sollten die Bewerber in der Weiterbildung sein. Die Stipendien werden an diejenigen vergeben, deren wissenschaftlicher Vortrag oder wissenschaftliches Poster für die Tagung angenommen wurde. Die DRG hat insgesamt zehn solcher Stipendien ausgelobt. Sie sind mit je 500,- Euro dotiert. Bei mehr Bewerbungen entscheidet das Jury-Committee der ICIS, das auch über die Annahme der Abstracts befindet. Die Stipendien sind nur insofern zweckgebunden, als dass sie für die Kosten eingesetzt werden soll, die in Zusammenhang mit der Kongressteilnahme stehen. Ansonsten ist der Stipendiat frei in der Wahl seiner Unterkunft, der Anreise etc. Das Stipendium soll als finanzieller Zuschuss eine zusätzliche Anerkennung des eingereichten Abstracts oder Posters darstellen.

Die AG Onkologische Bildgebung richtet am 30. September 2017 in Berlin eine eintägige Fortbildungsveranstaltung aus. Gibt es eine inhaltliche Verknüpfung bzw. bauen die Veranstaltungen aufeinander auf?

Die organisatorische Verknüpfung besteht einerseits im Tagungsort, an dem die Fortbildungsveranstaltung der AG Onkologische Bildgebung stattfindet, andererseits über die DRG, die auch die ICIS-Tagung unterstützt. Außerdem gibt es als Kombi- angebot einen finanziellen Rabatt für die Teilnahme beider Veranstaltungen. Dieses Angebot richtet sich an alle deutschen Radiologen, die sich für onkologische Bildgebung interessieren. Inhaltlich sind die Themen eng aufeinander abgestimmt. Bei der AG-Veranstaltung stehen die Tumorgruppen Prostata, Mamma und maligne Lymphome im Mittelpunkt. Alle drei Themen finden sich auch im ICIS-Programm wider, aber mit anderen Schwerpunkten. Wenn man beide Veranstaltungen besucht, ergibt sich damit wahrscheinlich ein runderes Gesamtbild.

\section{Und zum Schluss die Frage: Weshalb soll- te man die ICIS-Tagung 2017 in Berlin auf gar keinen Fall verpassen?}

Weil man einen kompletten Überblick über Bildgebung und Intervention bei seltenen und häufigen onkologischen Erkrankungen bekommt. Und wenn man sich als Radiologie teilweise oder auch intensiv mit der Bildgebung bei Krebspatienten beschäftigt, stellt die ICIS-Tagung sicherlich auf viele Jahre hin zumindest auf deutschem Boden die kompakteste Veranstaltung dar, um sein Wissen zu erweitern, zu festigen und konkreter anwenden zu können. 